\title{
A Resolução De Problemas No Ensino De Estatística No Ensino Fundamental: Contribuições Da Teoria Antropológica Do Didático E A Equivalência De Estímulos
}

\section{Solving problem in teaching statistics at elementary school: contributions of the anthropological theory of the didactic and the stimulus equivalence}

\author{
Ailton Paulo de Oliveira Júnior* \\ Universidade Federal do ABC \\ Natália Galvão Simão de Souza ${ }^{* *}$ \\ Universidade Federal do ABC \\ Nilceia Datori Barbosa ${ }^{* * *}$ \\ Universidade Federal do ABC
}

\begin{abstract}
Resumo
O objetivo do trabalho é apresentar a fundamentação teórica utilizada para a criação de problemas no processo ensino e aprendizagem de conteúdos estatísticos do $1^{\circ}$ ano do Ensino Fundamental, seguindo os princípios da Teoria Antropológica do Didático - TAD, na organização praxeológica didática e matemática (Estatística) e a Equivalência de Estímulos, para elaborar pequenas unidades de ensino, descrevendo um repertório simples a ser ensinado e progressivamente ir aumentando a complexidade. $\mathrm{Na}$ equivalência de estímulos apresentamos uma proposta para a fase de pré-teste considerando que o critério para avançar será de $100 \%$, ou seja, o aluno somente passará para a fase de ensino e teste de discriminações condicionais se acertar a todos os problemas propostos nessa fase. Também utilizaremos os princípios da Teoria Antropológica do Didático - TAD na organização praxeológica didática e matemática (Estatística), constituída de uma sequência de tarefas, que podem ser realizadas utilizando diversas técnicas justificadas pela tecnologia que se utiliza de teorias relacionadas à Estatística como objeto de estudo. Partindo desses pressupostos trazemos a elaboração de subtarefas abordando a necessidade da apreensão da nomenclatura e representação visual para tabela e a leitura de tabelas de colunas simples e coleta e organização de informações sugeridas pela Base Nacional Comum Curricular - BNCC. Acreditamos que utilizar a resolução de problemas como metodologia de ensino constitui-se uma forma interessante de apresentar conceitos básicos da Estatística, pois permitem que estes sejam apresentados de modo atrativo e favorecem a criatividade na elaboração de estratégias de resolução e busca de soluções.
\end{abstract}

Palavras-chave: Ensino de Estatística, Ensino Fundamental, Teoria Antropológica do Didático, Equivalência de Estímulos.

\footnotetext{
* Doutor e Pós-doutor em Educação (USP). Professor associado 2 da Universidade Federal do ABC, Santo André, São Paulo, Brasil. E-mail: ailton.junior@ufabc.edu.br.

${ }^{* *}$ Mestranda em Ensino e História das Ciências e Matemática da Universidade Federal do ABC, Santo André, São Paulo, Brasil. E-mail: natalia.galvao@ufabc.edu.br.

*** Mestranda em Ensino e História das Ciências e Matemática da Universidade Federal do ABC, Santo André, São Paulo, Brasil. E-mail: nilceiadatori@gmail.com.
} 


\begin{abstract}
The objective of this paper is to present the theoretical basis used for the creation of problems in the teaching and learning process of statistical contents of the 1st year of Basic Education, following the principles of the Anthropological Theory of the Didactic (TAD), in the didactic and mathematical praxeological organization ) and the Stimulus Equivalence, to elaborate small units of teaching, describing a simple repertoire to be taught and progressively increasing complexity. In the Stimulus Equivalence we present a proposal for the pre-test phase considering that the criterion to advance will be of $100 \%$, that is, the student will only go to the teaching and test phase of conditional discriminations if he agrees to all the problems proposed in this phase. We will also use the principles of the Anthropological Theory of the Didactic (TAD) in the didactic and mathematical praxeological organization (Statistics), made up of a sequence of tasks that can be performed using several techniques justified by the technology that uses theories related to Statistics as object of study. Based on these assumptions we bring the subtasks elaboration addressing the need for the apprehension of nomenclature and visual representation for table and the reading of tables of simple columns and collection and organization of information suggested by the National Curricular Common Base - BNCC. We believe that using problem solving as a teaching methodology is an interesting way to present basic concepts of Statistics, as they allow them to be presented in an attractive way and favor creativity in the elaboration of strategies for solving and finding solutions.
\end{abstract}

Keywords: Teaching Statistics, Elementary school, The Anthropological Theory of the Didactic, Stimulus Equivalence.

\title{
1 Introdução
}

Consideramos que quando se ensina através da resolução de problemas, auxilia-se os alunos a desenvolver sua capacidade de aprender a apreender, habituando-os a determinar por si mesmos respostas às questões que os inquietam, sejam elas questões escolares ou da vida cotidiana, ao invés de esperar uma resposta já pronta dada pelo professor ou pelo livro-texto.

Porém, não basta apenas ensinar a resolver problemas, mas incentivar o aluno a propor situações problema, partindo da realidade que o cerca, que mereçam dedicação e estudo.

Assim, esse trabalho tem como objetivo apresentar a fundamentação teórica utilizada para a criação de problemas no processo ensino e aprendizagem de conteúdos estatísticos do $1^{\circ}$ ano do Ensino Fundamental, seguindo os princípios da Teoria Antropológica do Didático TAD de Chevallard (1996) e Chevallard, Bosch e Gascón (2001), na organização praxeológica didática e matemática (Estatística) e a Equivalência de Estímulos (Sidman \& Tailby, 1982), para elaborar pequenas unidades de ensino, descrevendo um repertório simples a ser ensinado e progressivamente ir aumentando a complexidade.

Pretendemos trazer contribuições para o processo ensino e aprendizagem de estatística utilizando a Metodologia de Resolução de Problemas dos anos iniciais do Ensino Fundamental, apoiado na nova Base Nacional Comum Curricular-BNCC para o Ensino Fundamental (Brasil, 
2017), e no documento GAISE - Guidelines for Assessment and Instruction in Statistics Education (Diretrizes para Avaliação e Instrução em Educação Estatística (Franklin et al., 2005).

\section{Referencial Teórico}

Lopes (2008, p. 57) ressalta que a resolução de problemas é uma metodologia que pode servir ao desenvolvimento do trabalho com Estatística em sala de aula, pois da mesma forma que a Matemática, a Estatística se desenvolveu na história da humanidade através da resolução de problemas de ordem prática.

Apresentando a aplicação dessa metodologia, Vargas (2013) investigou a contribuição da Metodologia de Resolução de Problemas no ensino de Estatística para alunos do nono ano do Ensino Fundamental. O autor identificou que após a aplicação da atividade, os alunos evoluíram no conhecimento sobre Estatística, apropriaram-se de conceitos e perceberam o inestimável valor deles como ferramenta de leitura e compreensão da realidade, o que é um ponto de partida decisivo para a adoção de uma postura que busque transformações sociais e econômicas positivas na vida dos alunos e de seus familiares.

Também Neres e Cantanhêde (2016) investigaram a viabilidade do ensinoaprendizagem de estatística empregando a metodologia de Resolução de Problemas, sendo que os resultados revelaram que os alunos apresentaram bom desempenho na resolução dos problemas propostos.

Um aspecto importante a ser considerado na metodologia da resolução de problemas é trazido por Silva (2016) quando coloca ser visível a existência de uma grande confusão entre as definições de exercício e problema, ou seja, o exercício é uma atividade de treinamento no uso de alguma habilidade ou conhecimento matemático adquirido anteriormente pelo aluno, por exemplo, a aplicação de uma fórmula ou um algoritmo. E um problema refere-se a uma situação desafiadora, que exige do aluno criatividade, originalidade, reflexão e tomada de decisões, o que favorece a aquisição de experiências que o ajudará a tomar suas próprias decisões e pensar por si mesmo, ou seja, construir o seu próprio conhecimento matemático e descobrir suas próprias respostas.

Van de Walle (2009) também discute que um problema é qualquer tarefa ou atividade para a qual os estudantes não têm métodos ou regras prescritas ou memorizadas, nem a percepção de que haja um método específico para chegar à solução correta. Acrescentando um 
caráter subjetivo a essa questão, no contexto da metodologia aqui apresentada, consideramos que problema refere-se a tudo aquilo que não sabemos fazer, mas que estamos interessados em fazer.

Considerando ainda a prática, Fernandes e Santos Júnior (2017) investigaram de que maneira a resolução de problemas em situações de jogos, pode ser uma ferramenta de contribuição para o ensino e a aprendizagem dos conhecimentos matemáticos referentes à Estatística e a Probabilidade.

Dentre os principais pesquisadores no Brasil sobre a resolução de problemas, Onuchic e Allevato (2009) consideram que a aplicação de conteúdos estatísticos no ensino fundamental conforme recomendam os Parâmetros Curriculares Nacionais - PCN, devem ser realizados de forma crítica, com foco na leitura e interpretação de dados, e não apenas nos cálculos e na álgebra e a metodologia de ensino escolhida para alcançar essas metas é a de resolução de problemas. O método procura gerar debates, interação e descoberta pelos alunos, mediante uma postura de estímulo de parte do professor que definem como observação participante.

E internacionalmente, Gal (2002) aponta que os estudos estatísticos são ferramentas importantes para a formação de um cidadão capacitado a resolver situações-problema que estão presentes em seu cotidiano com melhor desempenho. Dessa forma, enfatiza que a alfabetização estatística está diretamente vinculada a cinco elementos cognitivos, a saber, habilidades de alfabetização, estatístico, matemático, conhecimento do contexto e questão crítica e, ainda, componente de disposição formado por posição crítica, convicção e atitudes.

Por fim, Batanero (2001), uma das principais referências mundiais, evidencia que em uma sociedade em constante mudança e imprevisível como a que vivemos, há insegurança sobre qual é a melhor forma de preparar os jovens e quais são os conteúdos que se deve ensinar. $\mathrm{O}$ que hoje nos parece essencial é que dedicamos grande parte do nosso tempo no processo ensino e aprendizagem pode ficar obsoleto em um tempo muito curto.

\section{Procedimentos Metodológicos}

Utilizamos como base teórica a TAD (Chevallard, 1996; Chevallard, Bosch, \& Gascón, 2001) para a elaboração dos problemas compostos por situações problema ou tipos de tarefa que identificaremos por $(T)$, constituída de uma sequência de subtarefas $(t)$, que podem ser realizadas utilizando diversas técnicas $(\tau)$ justificadas pela tecnologia $(\theta)$ que se utiliza da teoria Estatística $(\Theta)$ como objeto de estudo. 
Segundo Bittar (2017), o modelo praxeológico proposto para descrever qualquer atividade, matemática ou não, é composto por: tipo de tarefas T; técnicas $(\tau)$ que resolvem as tarefas desse tipo; tecnologia $(\theta)$ que justificam as técnicas e garantem sua validade, e, finamente, a teoria $(\Theta)$ que justifica a tecnologia. Esse quarteto praxeológico é denotado $[\mathrm{T}, \tau$, $\theta, \Theta]$, sendo que o bloco $[\mathrm{T}, \tau]$ é denominado de prático-técnico, ou bloco do saber-fazer; e o bloco $[\theta, \Theta]$ é denominado bloco tecnológico-teórico ou bloco do saber.

Juntamente com a TAD será considerado o documento GAISE, Franklin et al. (2005), focando no papel do conceito de variabilidade no processo de Resolução de problemas no Ensino de Estatística. Neste documento, é importante desenvolver o pensamento estatístico, utilizando dados reais, promovendo o uso da tecnologia e analisando os dados encontrados, para verificar a aprendizagem do aluno.

Tomaremos ainda como base metodológica a Equivalência de Estímulos (Sidman \& Tailby, 1982), fornecendo critérios operacionais, empiricamente verificáveis, para especificar comportamentos com características simbólicas. O modelo distingue relações entre pares associados (i.e., relações condicionais do tipo se..., então...) de relações de equivalência, potencialmente simbólicas.

Segundo Carmo e Galvão (2000), a Equivalência de Estímulos é um modelo teórico que permite prever que, para um indivíduo, um estímulo passa a pertencer a uma classe de estímulos equivalentes na qual estes são substituíveis uns pelos outros, a partir de relações condicionais arbitrariamente estabelecidas entre ele e um ou alguns membros dessa classe. Tal modelo é definido com base na presença de três propriedades extraídas da teoria dos conjuntos: reflexividade, simetria e transitividade.

A utilização da Equivalência de Estímulos em sua coleta de dados é composta pelas seguintes fases de pesquisa: (a) pré-teste; (b) ensino e teste de discriminações condicionais; (c) pós-teste. Neste trabalho, apresentamos problemas elaborados para a fase de pré-teste considerando que o critério para avançar será de $100 \%$, ou seja, o aluno somente passará para a fase de ensino e teste de discriminações condicionais se acertar a todos os problemas propostos nessa fase.

O procedimento experimental em experimentos de equivalência de estímulos pode variar de acordo com o emprego de diferentes protocolos. Fields, Reeve, Adams e Verhave (1991) distinguiram entre protocolos de treinamento simples para complexos, complexos para simples e simultâneos. 
O protocolo simultâneo envolve apresentações aleatórias das relações necessárias para estabelecer o controle de estímulo pré-requisito para o desempenho potencial de equivalência de estímulo. Isso geralmente é seguido por um bloco de testes separado que apresenta aleatoriamente testes de desempenho indicativos de equivalência de estímulos.

Além da possibilidade em utilizar diferentes protocolos, os experimentos de equivalência de estímulo podem ser distinguidos pelas estruturas de treinamento usadas para estabelecer as discriminações condicionais de pré-requisitos.

As três séries de estruturas de treinamento, quais sejam, de série linear (LS), de muitos para um (MTO) e de um para muitos (OTM) podem ser usadas para treinar as discriminações condicionais de pré-requisitos para responder de acordo com a equivalência de estímulos.

Embora nas análises originais de Murray Sidman se esperasse que as três estruturas de treinamento levassem ao mesmo resultado nos testes de equivalência (Sidman \& Tailby, 1982), estudos posteriores mostraram que a resposta de acordo com a equivalência de estímulo varia em função de diferentes estruturas de formação.

Portanto, nesse trabalho, levantamos a questão mais geral em considerar que o fracasso em estabelecer a resposta de acordo com a equivalência de estímulo é causada pelo participante experimental que não consegue executar as atividades de acordo com as contingências estabelecidas no treinamento básico de pré-requisito.

Para examinar os pré-requisitos (pré-teste), nosso experimento incluiu ensaios não reforçados de relações de linha de base aleatoriamente intercaladas entre testes de simetria, de transitividade e de equivalência global.

Segundo Sidman e Tailby (1982) a simetria mostra a reversão das funções condicionais e discriminatórias dos estímulos usados no treinamento, ou seja, dois elementos relacionados numa certa ordem, estão relacionados na ordem inversa, isto é, se $a \mathrm{R} b$, então $b \mathrm{R} a$. A transitividade refere-se a responder mostrando uma recombinação de estímulos relacionados no treinamento por membros de classes compartilhadas, ou seja, dois elementos relacionados a um elemento em comum, esses dois elementos devem estar relacionados entre si, ou seja, se $a \mathrm{R} b$ e $b \mathrm{R} c$, então $a \mathrm{R} c$.

O princípio de simetria significa que o aluno seja capaz de fazer reversível cada associação de elementos, por exemplo, caso a imagem do número 4 se associa com a palavra "quatro", a palavra "quatro" também se associa com a imagem do número 4 (Figura 1). 


\section{$4 \longrightarrow$ QUATRO \\ QUATRO $\longrightarrow 4$}

Fonte: Elaborado pelos autores.

Figura 1: Exemplo de uma relação de simetria na Equivalência de Estímulos no ensino de Números.

A simetria é uma relação que emerge a partir do treino de outra. Por exemplo, se ensinada a relação $\mathrm{AB}$, é possível inferir o surgimento da simetria quando o indivíduo consegue apresentar a "relação inversa" àquela que lhe foi ensinada, ou seja, a relação BA.

De Rose (1993) afirma que em uma relação simétrica, a validade da relação AB implicará necessariamente a validade da relação BA (Figura 2).


Fonte: Elaborado pelos autores.

Figura 2: Exemplo de relação de simetria (pareamento por identidade)

No princípio da transitividade o aluno deve ser capaz de intercambiar e manejar ideias associadas que compartilham um item comum, por exemplo, a Figura 3, mostra que se a maça $A$ é maior que a maça $B$, e a maça $B$ é maior do que a maça $C$, pode-se inferir que a maça $A$ também é maior do que a maça $\mathrm{C}$.



Fonte: Elaborado pelos autores.

Figura 3: Exemplo de uma relação de transitividade na Equivalência de Estímulos no ensino de Grandezas e Medidas.

Em relação à transitividade, Souza, Assis e Magalhães (2005, p. 297) afirmam que esta propriedade evidencia que "uma nova relação é formada a partir de duas anteriores nas quais há um estímulo comum". 
Portanto, para a ocorrência da transitividade devem ser ensinadas ao menos duas relações. Neste caso, se determinado estímulo A relaciona-se com B e B relaciona-se com C, então há transitividade se pudermos afirmar que $\mathrm{A}$ e $\mathrm{C}$ se relacionam.

Em outras palavras, em tendo sido treinadas duas relações (A1 B1 e B1 C1), havendo um elemento comum entre elas (no caso B1), emerge, sem treino, outra relação (A1C1).

Segundo Sidman (1986) é possível organizar testes combinados para que a simetria e a transitividade possam ser avaliadas simultaneamente e tal desempenho pode ser chamado de equivalência global.

Com o presente estudo pretende-se examinar a resposta dada a ausência de resposta de acordo com a equivalência de estímulos, considerando uma estrutura de treinamento LS (série linear) e o protocolo de treinamento simples para complexo, de forma a aumentar as chances dos participantes exibirem equivalência de estímulo.

\section{A Resolução De Problemas No Ensino De Estatística Para O $1^{\circ}$ Ano Do Ensino Fundamental}

Inicialmente temos como intenção que os alunos tenham conhecimento dos termos utilizados no ensino de estatística voltado ao letramento estatístico.

Segundo a Base Nacional Comum Curricular - BNCC (Brasil, 2017) no Ensino Fundamental, a articulação dos diversos campos da matemática, assim como Estatística e Probabilidade, precisa garantir que os alunos relacionem observações empíricas do mundo real a diferentes representações e associem essas representações a uma atividade matemática (conceitos e propriedades), fazendo induções e conjecturas.

Segundo Gal (2002), considerando os elementos estatísticos, o termo letramento referese à habilidade que os cidadãos devem ter para entender informações de natureza estatística que acessam em suas vidas diárias, e que define como a união das seguintes competências: (1) Interpretar e avaliar criticamente a informação estatística, argumentos apoiados por dados ou fenômenos estocásticos que as pessoas possam encontrar em diferentes contextos, incluindo os meios de comunicação, mas não limitados a eles; (2) Discutir ou comunicar suas opiniões sobre tais informações estatísticas, quando relevante.

As atividades curriculares elaboradas utilizando a resolução de problemas têm seu processo de criação considerando os conteúdos da proposta curricular da nova Base Nacional Comum Curricular - BNCC para os anos iniciais do Ensino Fundamental, Brasil (2017), de forma a possibilitar aos alunos a compreensão de conceitos básicos da Estatística (Quadro 1). 


\begin{tabular}{|l|l|l|l|}
\hline OBJETIVOS & $\begin{array}{l}\text { Leitura de tabelas e } \\
\text { de gráficos de } \\
\text { colunas simples. }\end{array}$ & $\begin{array}{l}\text { Registros pessoais } \\
\text { Coleta e organização } \\
\text { pe informações. } \\
\text { informações } \\
\text { coletadas. }\end{array}$ \\
\hline HABILIDADES de \\
$\begin{array}{l}\text { (EFO1MA21) Ler } \\
\text { dados expressos em } \\
\text { tabelas e em gráficos } \\
\text { de colunas simples. }\end{array}$ & $\begin{array}{l}\text { (EFO1MA22) Realizar pesquisa, envolvendo } \\
\text { interesse e universo de até 30 elementos, e } \\
\text { organizar dados por meio de representações } \\
\text { pessoais. }\end{array}$ \\
\hline
\end{tabular}

Fonte: Brasil (2017, p. 276-277).

Quadro 1: Objetivos e Habilidades dos conteúdos estatísticos propostos na nova Base Nacional Comum Curricular - BNCC do $1^{\circ}$ do Ensino Fundamental.

Neste trabalho, trazemos a elaboração de problemas abordando a representação visual e a nomenclatura de tabelas, a leitura de tabelas e de gráficos de colunas simples, e a ideia da coleta e organização de informações.

Considerando inicialmente um conhecimento mais simples utilizamos o princípio da simetria, o termo "tabela" (A) se associa com a representação visual que se constitui da mesma ideia de "tabela" (B) ou ao contrário, a representação visual que se constitui da ideia de "tabela" (B) está associado ao termo "tabela" (A) (Figuras 4 e 5).

\begin{tabular}{|c|c|c|c|c|c|}
\hline Tabela & $\begin{array}{l}\begin{array}{l}\text { Time de } \\
\text { futebol }\end{array} \\
\text { Corinthians } \\
\text { São Paulo } \\
\text { Santos } \\
\text { Palmeiras } \\
\text { Flamengo } \\
\text { Botafogo } \\
\end{array}$ & $\begin{array}{l}\begin{array}{l}\text { Número de } \\
\text { Votos }\end{array} \\
11111111 \\
11111 \\
111 \\
1111 \\
\end{array}$ & $\begin{array}{l}\begin{array}{l}\text { Time de } \\
\text { futebol }\end{array} \\
\text { Corinthians } \\
\text { São Paulo } \\
\text { Santos } \\
\text { Palmeiras } \\
\text { Flamengo }\end{array}$ & \begin{tabular}{|l|}
$\begin{array}{l}\text { Número de } \\
\text { Votos }\end{array}$ \\
1111|||| \\
IIIII \\
$11 \mid$ \\
IIII \\
\end{tabular} & \\
\hline & & & Botafogo & & \\
\hline
\end{tabular}

Fonte: Elaborado pelos autores.

Figura 4: Subtarefa 1 considerando a relação de simetria na Equivalência de Estímulos no ensino de Estatística.

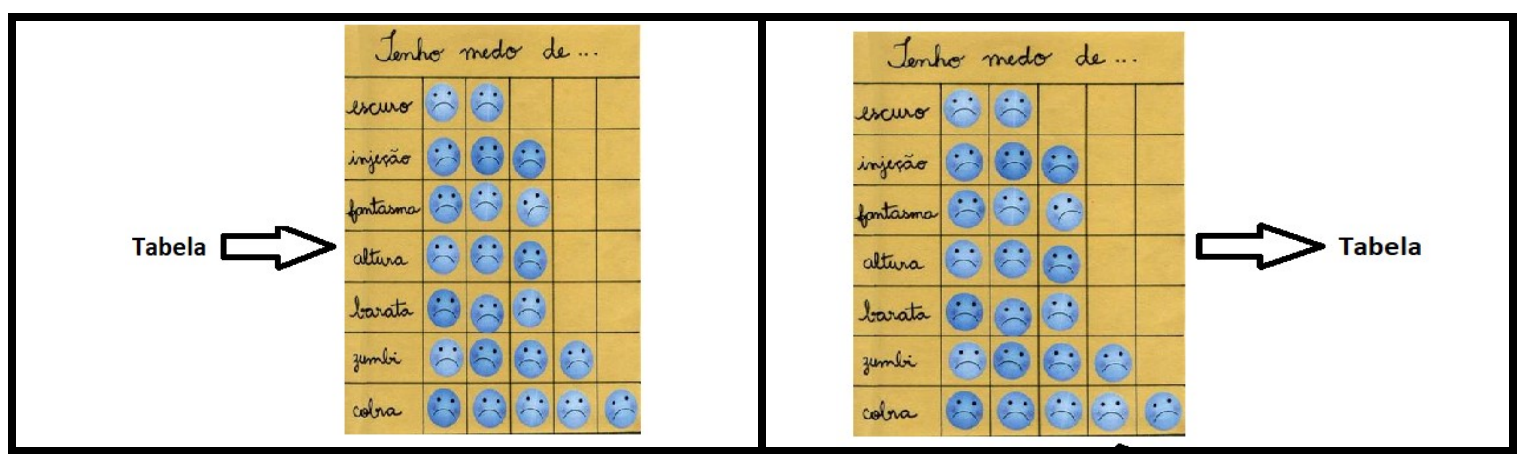

Fonte: Elaborado pelos autores.

Figura 5: Subtarefa 2 considerando a relação de simetria na Equivalência de Estímulos no ensino de Estatística.

Diz-se que ocorreu a simetria, figuras 4 e 5, pois há a identificação do termo "Tabela" associado à representação visual de "Tabela" representada com a sua idêntica representação (AB e BA). 
O objetivo didático para essa atividade é que o aluno consiga se apropriar do termo "tabela" que é a forma não discursiva de apresentação de informações, representadas por dados numéricos e codificações, dispostos em uma ordem determinada, segundo as variáveis analisadas de um fenômeno.

Existem várias regras para a apresentação de tabelas, porém, essas não devem ser rígidas. Muitas vezes a criatividade na sua montagem e edição é necessária para alcançar melhor comunicação.

Assim, tomando como base a TAD, a tarefa $\left(\mathrm{T}_{1}\right)$ consiste em associar a palavra "tabela" à representação visual de "tabela".

A técnica $\left(\tau_{1}\right)$, relaciona-se, respectivamente, à subtarefa 1 onde a palavra "tabela" à representação visual de uma tabela de colunas simples com representação numérica das frequências (figura 4) e à subtarefa 2 onde a representação visual de uma tabela de colunas simples com representação figural e lúdica se associa a representações numéricas (figura 5).

O par tecnologia/teoria $\theta_{1} \Theta_{1}$, que permite justificar e explicar a técnica $\left(\tau_{1}\right)$ pode ser descrita considerando Peça (2008) quando descreve as tabelas estatísticas, fazendo parte de uma linguagem universal, ou seja, é uma das formas de apresentação de dados para descrever informações, com o objetivo de produzir uma impressão mais rápida e viva do assunto em estudo, que podem ser vistos frequentemente ocupando lugar de destaque nos meios de comunicação, escrita e falada.

Ainda no pré-teste propomos atividades sobre a leitura de tabelas de colunas simples, associado à ideia da realização de uma pesquisa que convergem para a coleta dos dados e a sua organização.

Para Wainer (1992) é essencial que tenhamos ciência do tipo de perguntas tabulares que utilizaremos a fim de medir o nível de leitura que um indivíduo se encontra, a saber: (1) Nível básico; (2) Nível intermediário; (3) Nível avançado.

Consideramos que para o primeiro ano do Ensino Fundamental indicada pela nova BNCC, principalmente quando se considera a leitura de tabelas de colunas simples, levando ainda em consideração a coleta e organização de informações de registros pessoais, os alunos deverão ter domínio do nível básico paras as tabelas estatísticas onde somente são extraídas da tabela os dados que estão explícitos

Ainda consideramos aspectos do documento GAISE (Franklin et al., 2005) onde a resolução de problemas estatísticos é um processo investigativo que envolve quatro componentes: (1) Formular perguntas: Esclarece o problema e formula uma (ou mais) perguntas 
que podem ser respondidas com dados (informações). No caso do problema estabelecemos a seguinte pergunta de pesquisa: “Qual o tipo de gelatina que você mais gosta?”; (2) Coletar dados: Elabora um plano apropriado para coletar dados e emprega o plano para coletar os dados. No comando da questão é indicado que os alunos realizaram a pesquisa na Escola Estadual Natália Galvão; (3) Analisar: Seleciona métodos gráficos ou numéricos adequados e utiliza esses métodos para analisar os dados. A representação dos dados coletados é realizada por uma tabela simples em que são apresentados três tipos de gelatina e o número de alunos que preferem cada uma delas; (4) Interpretar os resultados: Interpreta a análise e relata a interpretação de acordo com a pergunta inicial ou provocadora do problema. Este aspecto será contemplado nas perguntas que serão realizadas.

A figura 6 apresenta problemas abordando a leitura de tabelas de colunas simples e a coleta e organização de informações tendo ainda como objetivo atender aos pressupostos da resolução de problemas para o ensino de estatística segundo o documento GAISE.

Foi realizada uma pesquisa na escola estadual Natália Galvão onde foi feita a seguinte pergunta: QUAL O TIPO DE GELATINA QUE VOCÊ MAIS GOSTA?

Para apresentar os dados coletados, os alunos elaboraram a tabela a seguir, que apresenta o tipo de gelatina que os alunos mais gostam.

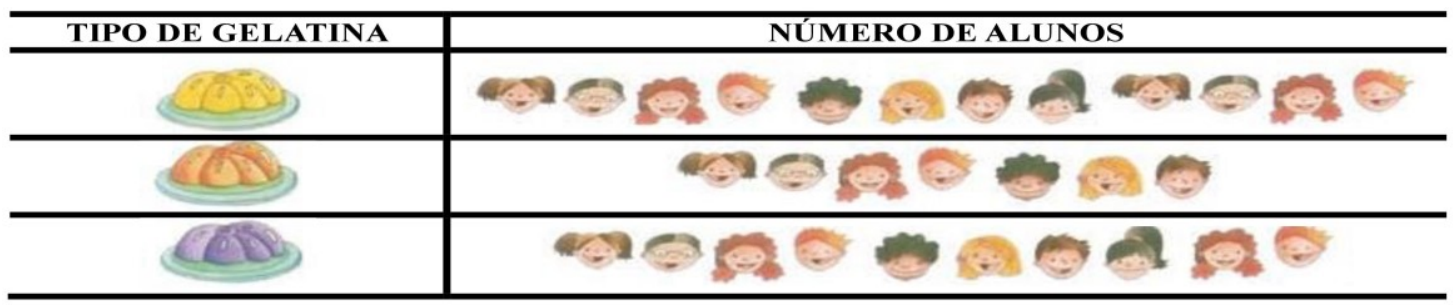

Para ajudar na análise dos dados da pesquisa, responda a seguinte pergunta:

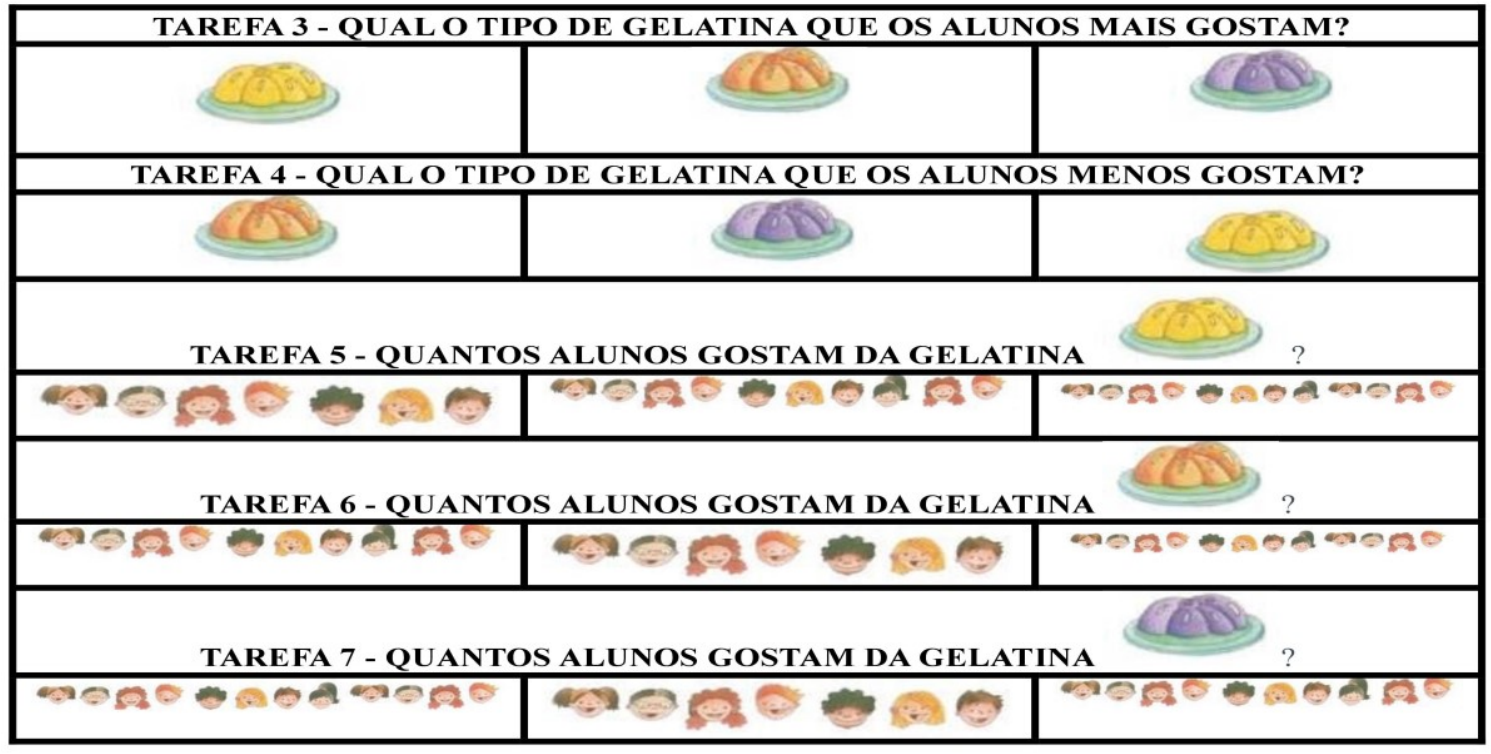

Fonte: Elaborado pelos autores.

Figura 6 :Tarefas 3 a 7 utilizando abordando a leitura dos dados apresentados em tabela estatística. 
Considerando a Equivalência de Estímulos e o currículo proposto pela nova BNCC, após selecionar, a partir do tipo de gelatina da pesquisa, aquela que os alunos mais gostam e a que menos gostam e o número de alunos que gosta de cada tipo de gelatina, consideramos que, pode-se considerar a tabela e determinar qual é a frequência absoluta que cada um dos tipos de gelatina aparece na tabela e relacionada à situação de pesquisa proposta.

Para produzir a formação de uma classe de equivalência, utilizamos discriminações condicionais entre dois tipos de possibilidades (A, B) onde A corresponde ao tipo de gelatina, ou seja, $\left(A_{1}, A_{2}, A_{3}\right)$, sendo respectivamente, gelatina de cor amarela, laranja e roxa; e $B$ corresponde ao número de alunos que preferem cada tipo de gelatina, ou seja, $\left(\mathrm{B}_{1}, \mathrm{~B}_{2}, \mathrm{~B}_{3}\right)$, sendo respectivamente, 12 alunos que preferem a gelatina de cor amarela, sete alunos que preferem a gelatina laranja e 10 alunos que preferem a gelatina roxa, para cada problema.

Portanto, temos as seguintes discriminações condicionais:

- A tarefa 3 indica $\left(\mathrm{A}_{1}, \mathrm{~A}_{2}, \mathrm{~A}_{3}\right)$;

- $\quad$ tarefa $4\left(\mathrm{~A}_{2}, \mathrm{~A}_{3}, \mathrm{~A}_{1}\right)$;

- A tarefa 5 indica $\left(\mathrm{B}_{2}, \mathrm{~B}_{3}, \mathrm{~B}_{1}\right)$;

- A tarefa 6 indica $\left(\mathrm{B}_{3}, \mathrm{~B}_{2}, \mathrm{~B}_{1}\right)$;

- A tarefa 7 indica $\left(\mathrm{B}_{1}, \mathrm{~B}_{2}, \mathrm{~B}_{3}\right)$.

Considerando a TAD, as Tarefas $\left(\mathrm{T}_{3}\right.$ e $\left.\mathrm{T}_{4}\right)$ consistem, respectivamente, em determinar qual o tipo de gelatina que os alunos mais gostam e a que menos gostam, a partir da apresentação dos três tipos de gelatina que a pesquisa indicou como aquelas que os alunos consomem. A gelatina de cor amarela é a que mais gostam, pois representa o maior número ou frequência de alunos. E a gelatina de cor laranja a que menos gostam, representando o menor número ou frequência de alunos.

A técnica $\left(\tau_{2}\right)$ para resolver as tarefas $\mathrm{T}_{3}$ e $\mathrm{T}_{4}$ pode ser:

(1) A correspondência de termo a termo entre as coleções de estudantes que gostam de cada tipo de gelatina. De modo que quando temos duas coleções e estamos associando "um elemento" de uma coleção com "um elemento" de outra coleção e acontece que há "um ou vários elementos" da primeira coleção aos quais não podemos atribuir "um único elemento" da segunda seleção porque todos os elementos deste já possuem um elemento do primeiro atribuído, então pode-se dizer que a primeira coleção é maior que a segunda. 
Consequentemente, também pode ser dito que a segunda coleção é menor que a primeira.

(2) Contar as coleções de alunos que gostam de cada tipo de gelatina e comparar os números obtidos de cada coleção após a contagem, obtendo qual é o maior número e qual é o menor. Assim, a coleção com o maior número será a maior coleção e a coleção com o menor número será a menor.

As tarefas $\left(\mathrm{T}_{5}\right.$ a $\left.\mathrm{T}_{7}\right)$ consistem em determinar o número de alunos que optaram por cada um dos tipos de gelatina que os alunos declararam gostar na pesquisa. No caso, são doze alunos ou a terceira opção que gostam da gelatina de cor amarela (abacaxi); sete alunos ou a segunda opção que gostam da gelatina de cor laranja (laranja); e dez alunos ou a terceira opção que gostam da gelatina de cor roxa (uva). Buscamos alternar a ordem das opções nas três tarefas para gerar diferentes ordenamentos.

A técnica $\left(\tau_{3}\right)$ que responde às tarefas $T_{5}$ a $T_{7}$ corresponde à cardinalidade ao estabelecer que o último número utilizado na contagem de um conjunto indica o número de elementos dele (numerosidade). Reflete-se a indicação do número correto de um conjunto sem contar quando este conjunto foi previamente contado, ou seja, é respondido com a cardinalidade da primeira contagem. Deve-se contar o número de alunos que gostam de cada tipo de gelatina (amarela, laranja e uva) para identificar quantos são e associar à preferência por cada tipo de gelatina.

A tecnologia $\theta_{1}$, que permite justificar e explicar as técnicas $\left(\tau_{2}\right.$ e $\left.\tau_{3}\right)$ pode ser descrita considerando Duval (2002) quando descreve que a leitura e a interpretação de gráficos e tabelas é considerado por muitos como sendo simples devido a sua organização e a rapidez de consulta, porém essa leitura e interpretação não se dá de forma simples, pois precisa ativar todas as funções cognitivas. Em relação às tabelas a função identificação é a mais utilizada, devido à visualização dos dados de forma separada.

A teoria $\Theta_{1}$ que explica e justifica a tecnologia $\theta_{1}$ é fundamentada em Duval (2003), quando diz que o estudo de gráficos e tabelas deve ser pautado no trânsito entre diferentes tipos de registros, pois desta forma, proporciona-se a visualização de um mesmo objeto matemático sob diferentes formas, levando-se alunos ao não "enclausuramento de registros". Este último fato leva o indivíduo a ter um pensamento restrito a novas possibilidades.

A seguir são apresentadas tarefas focadas no conceito de variabilidade, figura 7, apoiados no documento GAISE, para que os alunos em diferentes situações se apropriem do conceito de variabilidade pela análise de uma tabela. Consideramos que deve-se agregar o estudo do conceito de variabilidade a partir da representação de dados via tabela. 


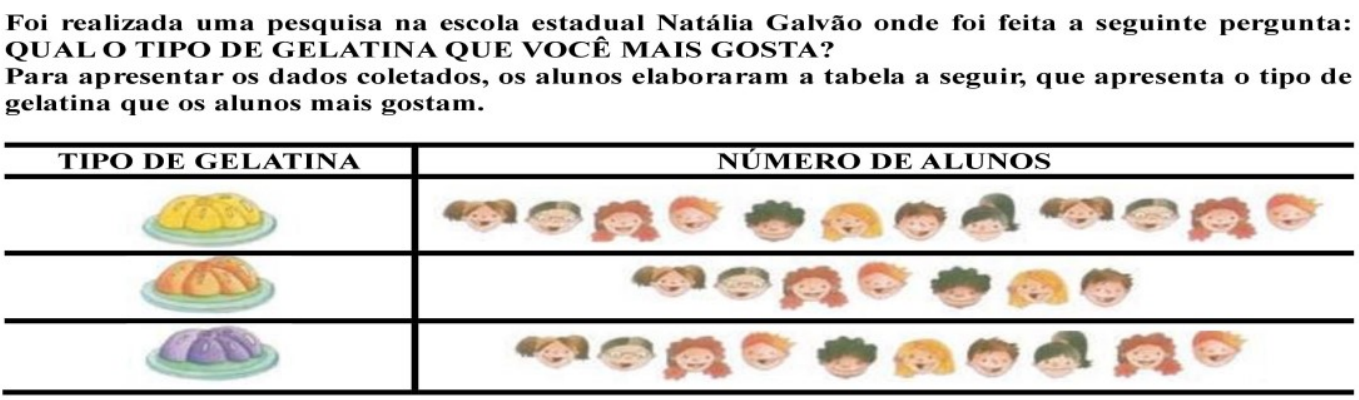

Para ajudar na análise dos dados da pesquisa, responda a seguinte pergunta:

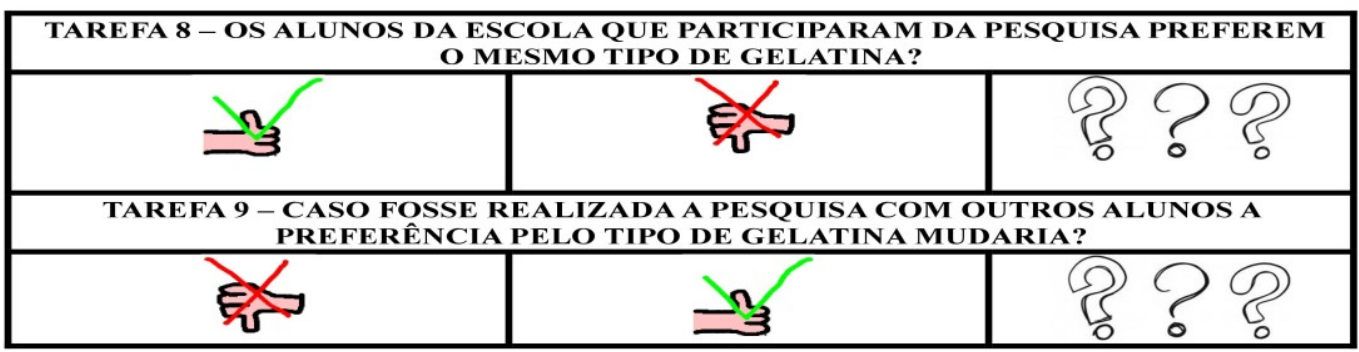

Fonte: Elaborado pelos autores.

Figura 7: Tarefas 8 e 9 abordando o conceito de variabilidade a partir de uma tabela estatística.

O conceito de variabilidade está relacionado com muitas ideias estatísticas fundamentais, sendo que Garfield e Ben-Zvi (2008) observaram que a compreensão das ideias de dispersão e variabilidade dos dados é componente chave para a compreensão do conceito de distribuição e é essencial para fazer inferências estatísticas.

Para produzir a formação de uma classe de equivalência, discriminações condicionais entre o tipo de possibilidade (C) que corresponde ao tipo de concordância em relação ao problemas proposto ao tipo de gelatina, ou seja, $\left(\mathrm{C}_{1}, \mathrm{C}_{2}, \mathrm{C}_{3}\right)$, sendo respectivamente, concordar com a situação proposta, não concordar com a situação proposta e não saber responder.

Portanto, temos as seguintes discriminações condicionais:

- A tarefa 8 indica $\left(\mathrm{C}_{1}, \mathrm{C}_{2}, \mathrm{C}_{3}\right)$;

- A tarefa $9\left(\mathrm{C}_{2}, \mathrm{C}_{1}, \mathrm{C}_{3}\right)$.

Considerando a TAD na organização praxeológica matemática (estatística), no caso da Tarefa $8\left(\mathrm{~T}_{8}\right)$ a resposta seria "Não", pois há um número diferenciado de opções, ou seja, variação na preferência pelos tipos de gelatina. E a Tarefa 9 ( $\left.\mathrm{T}_{9}\right)$ a resposta seria “Talvez", pois seria muito difícil que o resultado com outros alunos fosse o mesmo, também caracterizando variação.

A técnica $\left(\tau_{4}\right)$ que responde as tarefas $\mathrm{T}_{8}$ e $\mathrm{T}_{9}$ residem em identificar se existem fontes 
diferentes de variabilidade nos dados considerando que repetidas medições de uma mesma característica ou opinião do mesmo indivíduo pode variar.

O par tecnologia-teoria $\left(\theta_{2}, \Theta_{2}\right)$ que permite justificar e explicar a técnica $\left(\tau_{5}\right)$ é sustentado por Konold e Pollatsek (2004) recomendando que o centro de análise de dados (sinal) e variabilidade (ruído) seja considerado em conjunto, porque ambas as ideias são necessárias para dar sentido aos dados.

Além disso, para Watson, Callingham e Kelly (2007) a compreensão da variabilidade está relacionada com a percepção de incerteza, ou seja, com as mudanças e valores atípicos esperados e inesperados que os dados apresentam.

\section{Considerações Finais}

A organização praxeológica seguiu o princípio da Teoria Antropológica do Didático que situa a atividade matemática dentro do conjunto das atividades humanas e das instituições sociais. A proposta dessa teoria é que as práticas que os professores devem desenvolver, em sala de aula, devam ocorrer a partir de situações problemáticas que envolvam as especificidades das áreas relacionadas às condições sociais em que o estudante esteja inserido. Para isso, apresentou a noção de praxeologia, que significa, em sua essência, a tentativa de encontrar uma ou mais formas de resolver questões problemáticas, regularmente e com sucesso, no caso, conteúdos estatísticos para o primeiro ano do Ensino Fundamental, especificamente o ensino de tabelas estatísticas, a partir da Base Nacional Comum Curricular.

Consideramos ainda que os problemas propostos no presente estudo permitem concluir que o estabelecimento de relações de equivalência entre as diferentes formas de apresentação de problemas estatísticos pode ser relevante para melhorar o desempenho de alunos do primeiro ano do Ensino Fundamental na resolução de problemas, uma vez que a progressiva apreensão de conceitos básicos da estatística reduz o efeito de variáveis que tornam maior a complexidade da sucessão de habilidades necessárias para o domínio da leitura de tabelas estatísticas a partir de coleta de dados reais.

E, por fim, estabelecer relações de equivalência entre diferentes formas de apresentação dos problemas estatísticos, tendo o cuidado de variar situações do cotidiano do aluno, pode ser uma maneira de o professor levar esse a aprender que o comportamento (estratégia de resolução) apresentado em determinada situação pode ser usado em situações que são semelhantes, isto é, resolver com a mesma estratégia problemas que tem mesma forma 
(estrutura), e aprender que as mesmas estratégias são aplicáveis em situações nas quais os mesmos problemas são apresentados em diferentes formatos (estruturas diferentes).

\section{Referências}

Batanero, C. (2001). Didáctica de la Estadística. Granada: Grupo de Investigación em Educación Estadística. Disponível em https://www.ugr.es/ batanero/pages/ARTICULOS/didacticaestadistica.pdf.

Barros, R. S. (1996). Análise do comportamento: da contingência de reforço à equivalência de estímulos. Caderno de Textos de Psicologia, 1(1), 7-14.

Bittar, M. (2017). A Teoria Antropológica do Didático como ferramenta metodológica para análise de livros didáticos. Zetetiké, 25(3), 364-387.

Brasil. (2017). Base Nacional Comum Curricular: Educação é a base. Ministério da Educação, Brasília. http://basenacionalcomum.mec.gov.br/images/BNCC 20dez site.pdf.

Carmo, J. S., \& Galvão, O. G. (2000). Aquisição do conceito de número em crianças préescolares através do ensino de relações condicionais e generalização. In: Carmo, J. S., Silva, L. C. C., \& Figueiredo, R. M. E. (Org.). Dificuldades de aprendizagem no ensino de leitura, escrita e conceitos matemáticos (pp. 50-87). Universidade da Amazônia, Belém.

Chevallard,Y. (1996). Conceitos fundamentais da Didáctica: perspectivas trazidas por uma abordagem antropológica. In: BRUN, J. Didáctica das Matemáticas. Lisboa: Horizontes Pedagógicos.

Chevallard, Y., Bosch, M., \& Gascón, J. (2001). Estudar Matemáticas: o elo perdido entre o ensino e a aprendizagem. Porto Alegre: Artes Médicas.

De Rose, J. C. C. (1993). Classes de estímulos: Implicações para uma análise comportamental da cognição. Psicologia: Teoria e Pesquisa, 9(2), 283-303.

Duval, R. (2002). Comment analyser le fonctionnemment représentationnel des tableaux et leur diversité? In: Séminaires de Recherche "Conversion et articulation des représentations". Vol II. Éditeur Raymond Duval, IUFM Nord-Pas de Calais.

Duval, R. (2003). Registro de representações semióticas e funcionamento cognitivo da compreensão em matemática. In: Machado, S. D. A. (Org.). Aprendizagem em Matemática: registro de representação semiótica (pp 11- 33). Campinas, São Paulo: Papirus.

Fernandes, R. J. G., Santos Júnior, G. (2017). Ensino de Estatística e de Probabilidade para os anos iniciais de escolarização: uma proposta para trabalhar resolução de problemas em contextos de jogos. Boletim de Educação Matemática - BoEM, 5(9), 62-80. 
Fields, L., Reeve, K. F., Adams, B. J., \& Verhave, T. (1991). The generalization of equivalence relations: A model for natural categories. Journal of the Experimental Analysis of Behavior, $55,305-312$.

Franklin, C., Kader, G., Mewborn, D. S., Moreno, J., Peck, R., Perry, M., \& Scheaffer, R. (2005). A curriculum framework for K-12 statistics education. GAISE report. American Statistical Association. Disponível em www.amstat.org/education/gaise/.

Gal, I. (2002). Adult's statistical literacy: meanings, components, responsibilities - appears. International Statistical Review, 70(1), 1-33.

Garfield, J.; Ben-Zvi, D. (2008). Developing Students' Statistical Reasoning: Connecting Research and Teaching Practice. Springer.

Konold, C., \& Pollatsek, A. (2004). Conceptualizing an average as a stable feature of a noisy process. In D. Ben-Zvi, \& J. Garfield (Eds.). The challenge of developing statistical literacy reasoning and thinking (pp. 169-199). Dordrecht: Kluwer.

Lopes, C. A. E. (2008). O Ensino da Estatística e da Probabilidade na Educação Básica e a Formação dos Professores. Caderno CEDES, 28(74), 57-73.

Neres, R. L., \& Cantanhêde, R. D da S. (2016). Ensinar e aprender estatística por meio de resolução de problemas. Revista de Educação, Ciências e Matemática, 6(1), 59-77.

Onuchic, L. R., \& Allevato, N. S. G. (2009). Ensinando Matemática na sala de aula através da Resolução de Problemas. Boletim GEPEM, 55, 1-19.

Peça, C. M. K. (2008). Análise e interpretação de tabelas e gráficos estatísticos utilizando dados interdisciplinares. Programa de Desenvolvimento Educacional, UTFPR, Paraná. Disponível em http://www.diaadiaeducacao.pr.gov.br/portals/pde/arquivos/1663-8.pdf.

Sidman, M., \& Tailby, W. (1982). Conditional discrimination vs matching to sample: an expansion of the testing paradigm. Journal of the Experimental Analysis of Behavior, 37, $5-22$.

Sidman, M. (1986). Functional analysis of emergent verbal classes. In: Thompson, T.; Zeiler, M. D. (Orgs.). Analysis and Integration of Behavioral Units (pp. 213-245). Hillsdale, N. J.: Erlbaum.

Silva, V. F. (2016). A resolução de problemas: concepções evidenciadas na prática e no discurso de professores de matemática do Ensino Fundamental. Anais do 10 Simpósio Linguagens e Identidades da/na Amazônia Sul-Ocidental: Trânsitos pós-coloniais e de colonialidade de saberes e sentidos, Universidade Federal do Acre, Acre.

Souza, R. D. C., Assis, G. J. A., \& Magalhães, P. G. S. (2005). Emergência de relações numéricas sob controle condicional em crianças surdas, Psicologia: Teoria e Pesquisa, 21(3), 297-308.

Van De Walle, J. A. (2009). Matemática no Ensino Fundamental: formação de professores e aplicação em sala de aula. Porto Alegre: Artmed. 
Vargas, G. G. B. de. (2013) A metodologia da resolução de problemas e o ensino de estatística no nono ano do ensino fundamental. Dissertação de Mestrado em Ensino de Matemática, Mestrado Profissionalizante em Ensino de Física e de Matemática, Centro Universitário Franciscano de Santa Maria.

Wainer, H. (1992). Understanding graphs and tables. Educational Researcher, 21(1), 12-14.

Watson, J. M., Callingham, R. A., \& Kelly, B. A. (2007). Students' appreciation of expectation and variation as a foundation for statistical understanding. Mathematical Thinking and Learning, 9(2), 83-130.

Submetido em: 22/03/2019

Aceito em: 08/05/2019 